\title{
Relaxing to Three Dimensions
}

\section{Citation}

Karch, Andreas, and Lisa Randall. 2005. "Relaxing to Three Dimensions." Physical Review Letters 95 (16). https://doi.org/10.1103/physrevlett.95.161601.

\section{Permanent link}

http://nrs.harvard.edu/urn-3:HUL.InstRepos:41417297

\section{Terms of Use}

This article was downloaded from Harvard University's DASH repository, and is made available under the terms and conditions applicable to Other Posted Material, as set forth at http:// nrs.harvard.edu/urn-3:HUL.InstRepos:dash.current.terms-of-use\#LAA

\section{Share Your Story}

The Harvard community has made this article openly available.

Please share how this access benefits you. Submit a story.

Accessibility 
August 26, 2018

\title{
Relaxing to Three Dimensions
}

\author{
Andreas Karch \\ Department of Physics, University of Washington, Seattle, WA 98195, USA \\ Lisa Randall \\ Department of Physics, Harvard University, Cambridge, MA, 02138, USA
}

\begin{abstract}
We propose a new selection principle for distinguishing among possible vacua that we call the "relaxation principle." The idea is that the universe will naturally select among possible vacua through its cosmological evolution, and the configuration with the biggest filling fraction is the likeliest. We apply this idea to the question of the number of dimensions of space. We show that under conventional (but higher-dimensional) FRW evolution, a universe filled with equal numbers of branes and antibranes will naturally come to be dominated by 3-branes and 7-branes. We show why this might explain the number of dimensions that are experienced in our visible universe.
\end{abstract}

Introduction: No fundamental physical principle singles out three dimensions. Yet three dimensions of space clearly has a special status. The obvious question is why.

There have been several attempts to address this question, particularly in the context of string theory. Probably the idea that has received the most attention is the Brandenberger-Vafa suggestion [1]. They argue that four spacetime dimensions are singled out because the world sheet of a string occupies two dimensions and in four dimensions two two-dimensional worldsheets will intersect. Their argument is that unless the worldsheets can intersect, nothing dilutes the winding modes of strings. And if nothing dilutes the winding modes, the initially very small dimensions would never grow large. However, if there are four (or fewer) spacetime dimensions, strings can shed their windings so that the dimensions can grow.

This is an interesting scenario, but has several questionable aspects. One is that it critically relies on poorlyunderstood dynamics at the Planck scale. Another is that it requires a resolution of the moduli problem, and, more importantly, relies on simple toroidal compactification. But the most problematic feature of this solution is that it relies on strings being the sole important objects in string theory, whereas we now know that branes also play a critical role. Some work has been done on addressing this concern, see in particular 2].

Another more recent suggestion by [] $]$ was also made in the context of string theory with compact dimensions. They suggest another reason that four spacetime dimensions are special. They point out that $4+4<10$ and that this not true for any larger integer. Their argument was that the worldvolume of 3-branes would NOT intersect whereas the worldvolume of any larger branes would. Their argument was that larger branes can therefore unwind, whereas 3-branes would survive. Their suggestion is very interesting but has some technical problems. In particular, the unwinding mechanism they suggest is flawed-the branes would generally merge to form new (p,q)-type branes. The same numerical fact has also been used in [4], where the universe is created via a sequence of brane/antibrane annihilations. The observa- tion that $4+4<10$ is of course robust. Here we will use this numerical fact in an entirely different context, with NO compact dimensions.

Branes and localization have opened up an entirely new way of approaching higher-dimensional cosmology, since it is possible that both gravitational and nongravitational forces can be localized on 3-branes. Yet very few cosmological studies have been done that exploit this idea. We now know that the apparent number of dimensions isn't necessarily the same as the number of dimensions that actually exists, so it is reasonable to think the initial evolution of the universe might be higher-dimensional.

We invoke higher-dimensional cosmology in conjunction with what we call the "relaxation principle." The idea is that there are many conceivable vacua, as would be described, for example, in a landscape scenario. But rather than invoking the anthropic principle to chose among them, we suggest that the ones that are favored are those that dominate in a consistent stable cosmological scenario. One attempt in this direction in the context of the cosmological constant was given in [5].

In the context of brane gas dynamics, we assume that the branes with the biggest filling fraction in the endpoint of the universe's cosmological evolution are the most likely branes to be relevant to the state in which we live. String theory does indeed indicate there could be multiple vacua. This makes it especially worthwhile to investigate alternative selection principles. It is possible that dynamics, rather than static criteria, determines the vacuum in which we live.

In this letter we show that under some very general and plausible assumptions about brane dynamics in ten dimensions, branes with other numbers of dimensions will be diluted relative to the 3-branes and 7-branes. In our approach to cosmological evolution, we assume the number of dimensions is fixed but that the number of dimensions we see is determined by brane dynamics. We make a general ansatz that the universe is initially filled with branes of all sizes and that the stress-energy on the branes dominates the cosmological evolution. We then let the universe expand and look for a consistent solu- 
tion. We find the only stable evolution under reasonable general assumptions is into a universe dominated by 3branes and 7-branes. As in previous work, we will use the fact that 3-branes will be the largest objects whose self-intersections will not significantly reduce their density (since $4+4<10$ ).

Recent work on cosmic strings proceeds along similar lines to the analysis we present below. That work assumes three large dimensions whereas ours assumes nine. Our broader framework might be more appropriate in string theory now that we know that branes play a critical role. However, strings could play an important role along the lines of [ $[$ ] once the universe has settled into an effective four-spacetime-dimensional theory-that is, once all the other branes have diluted and gravity has localized on the brane intersection.

FRW Universe in higher dimensions: Starting with the $n+1$ dimensional Robertson Walker metric

$$
d s^{2}=-d t^{2}+a^{2}(t) d \Sigma_{k}^{2}
$$

with $n$ dimensional maximally symmetric spatial geometry $\Sigma_{k=-1,0,1}$, the Friedmann equations dimensions read:

$$
H^{2}=-\frac{k}{a^{2}}+\frac{16 \pi G_{N}}{n(n-1)} \rho
$$

Energy conservation demands

$$
\frac{\dot{\rho}}{\rho}=-n(1+w) \frac{\dot{a}}{a}
$$

where $w$, as usual, determines the equation of state of the matter system, $p=w \rho$. If the right hand side of (2) is dominated by a component of given $w$ we can solve for the time dependence of $a$ and $\rho$ in that era:

$$
\rho \sim a^{-n(1+w)} \rightarrow t \sim a^{\frac{n}{2}(w+1)}
$$

A few typical values of $w$ are $w=0$ for pressureless dust, $w=1 / n$ for radiation with traceless stress tensor, a cosmological constant has $p=-\rho$ and hence $w=-1$ in any $n$. Note that $w_{\text {crit. }}=-\frac{n-2}{n}$ sets the borderline between acceleration and deceleration. More interesting for us are the $w$ values of networks of topological defects, also known as brane gases. A string has $\rho=-p$ like a cosmological constant, but only one component of $p$ is non-zero, so with strings in random directions in $n=3$ spatial dimensions the average $p$ is going to be $-\frac{1}{3} \rho$. In the same way one can argue that a $d$-brane with a $d+$ 1 dimensional worldvolume in $n$ spatial dimensions has $w=-\frac{d}{n}$. For a non-interacting gas of $d$-branes according to (4) the energy density goes as $\rho_{d}^{n i} \sim a^{d-n}$; the volume of the brane goes as $a^{d}$, but the volume of space goes like $a^{n}$ so the energy density goes as $a^{d-n}$.

However, if the branes can self-intersect we expect them to decay and hence to dilute much faster. In the familiar case of $d=1$ strings in $n=3$ spatial dimensions, the decay mechanism is that strings that intersect spawn loops of closed string which then decay by emitting gravity waves, for a review see [6]. To see how the energy density of such a self-intersecting brane network behaves as a function of time one can follow the same very general line of logic that is usually applied for cosmic strings: assuming that the decay processes happen at the maximum efficiency allowed by causality, the network at any time looks the same when viewed at the horizon scale $t$. This is often referred to as the scaling solution. The total length of string within a horizon volume is hence some number times $t$. Similarly the total area of a two-dimensional membrane is some number times $t^{2}$ and the total volume of $d$-brane some number times $t^{d}$. The horizon volume is just given by $t^{n}$, hence $\rho_{d}^{i} \sim t^{d-n}$. In order to determine which type of defect is going to dominate the energy density, we need to know which defects will interact and which not. We assume that the generic situation is that any defects that can find each other will interact and trigger some decay mechanisms that will work with an efficiency only limited by causality. The efficiency with which branes and antibranes that find each other annihilate is a critical question that we would like to see checked in future work.

The question of whether branes can generically find each other depends only on their dimensionality. In $n+1$ dimensions generic (that is no parallel directions) $d$-branes with $2 d \geq n$ intersect at all times over a $2 d-n$ brane. For $2 d=n-1$ (e.g. strings in $n=3$ ), they intersect over a (-1) brane, that is they collide at an instant. $d$-branes with $2 d \leq n-2$ on the other hand generically do not find each other. For $n=3$ we reproduce the well known results that monopoles generically do not find each other, while cosmic strings and domain walls do. For the case $n=9$, which is the critical dimension of superstring theory, we see that branes with $d \leq 3$ will not find each other, while generic branes with $d \geq 4$ at least intersect at an instant in time. This simple counting was already proposed in [3] as a possible mechanism to select a 4-dimensional world, alas in the context of torus compactifications where the branes have conserved winding charges and hence generically cannot annihilate.

We also note an additional feature of branes that affects their density. Brane-antibrane pairs generically contain a tachyon. In the case of 9-branes, that tells us that 9-branes will generically annihilate since they overlap completely at all times. So 9-branes will not have energy and number density that scales as above, but will have zero density for all times.

The case of 8-branes is trickier. It might be that 8branes never exist since it is possible that only even or odd dimensional branes exist. This is true for D-branes in type II string theory. It would also be true of the tachyon is a complex scalar and lower dimensional branes are produced as defects in higher-dimensional-brane annihilation. This is analogous to the reasoning in [6], for example, where it was argued that strings but not domain walls might be present in the cosmos (domain walls give exactly the same problem in four-dimensions that 
8-branes give us here). Furthermore, another feature of string theory might affect 8-branes. In flat space, a static 8-brane/anti-8-brane configuration cannot have branes that are very far from each other or else spacetime breaks down. If this is also true for dynamical systems in arbitrary backgrounds, it would mean that 8-branes are also expected to have number density less than $t^{8-n}$.

We now turn to lower-dimensional branes. This is where the significance of three dimensions becomes apparent. Were there no brane intersections, the higherdimensional branes would dilute much more slowly. However, the intersections change the dilution so that lowerdimensional and higher-dimensional branes can compete. By assuming that if the branes intersect, they will annihilate and dissipate their energy at a rate determined by causality, we can compare the densities of the branes of different dimensionalities.

For $d \leq 3$, the branes dilute as $a^{d-n}$, whereas for higher $d$, they scale as $t^{d-n}$. Among the branes with $d \leq 3$, it is clear that the 3 -branes will dilute the least. To resolve the competition between $d \leq 3$ and higher dimensional branes, we need to know the relation between $a$ and $t$, or alternatively the $w$ of the dominant fluid component. For any $w<w_{\text {crit }}=-\frac{n-2}{n}$, (44) tells us that $t$ indeed grows slower than $a$. One option is to take the 3-brane energy density to dominate, $w=-\frac{1}{3}$, and to see if this is a self consistent solution. The resulting time-dependence of the scale factor is $a \sim t^{\frac{1}{3}}$, so it is clear that only 7 branes and 8 -branes can compete with 3 -branes. In fact, 7 -branes dilute at the same rate and 8-branes at a slower rate. For the reasons given earlier, it is reasonable to assume that 8-branes don't exist.

Really since we have assumed constant and equal energy densities, the energy density represents filling fraction in the universe. That is, since 3-branes and 7-branes dominate the energy density and hence the evolution of the ten-dimensional universe, they also dominate the filling-fraction of the universe. Therefore, a reasonable hypothesis would be that they are the most likely place for our universe to reside.

The result that 3-branes will eventually dominate is robust even if we start with a different fluid component driving the expansion. 3-branes have $w=-\frac{1}{3}$. If the universe is dominated by $w>-\frac{1}{3}$ (like $w=0$ dust or $w=\frac{1}{9}$ radiation) all that happens is that the 7 -branes can no longer keep up. For $w>\frac{1}{3} 3$-branes always dominate (assuming 9-branes annihilate). Note that this in particular implies that brane fluctuations and bending get washed out. The bending of the branes can be thought of as a massless scalar living on the worldvolume of the branes. Its gradient energy acts like a $w=1$ fluid and will hence be subdominant. So independent of which component dominates early on, a universe with 3-branes and 7-branes will be the only fixed point of the evolution. For the rest of the paper we restrict our attention to this universe filled with 3-branes and 7-branes.

Applications: In the previous section we have shown that the dynamics of brane networks will lead to a $10 \mathrm{~d}$ universe dominated by 3- and 7-branes. This calculation relied only on some simple dynamical assumptions about an expanding ten-dimensional universe, which should be explored further in the future. First was a homogenous and isotropic 10d universe, which is really a statement about the initial conditions we assume. The precise evolution we used depends on initial conditions and could be accounted for by a stage of ten-dimensional inflation. We also assumed that any brane and antibrane that intersects will annihilate, which also merits further investigation in the future. Finally, we need the dilaton to be stabilized. It is known that in the presence of an evolving dilaton the analysis of brane decay will be significantly altered [7, 8].

We now want to consider the physical implications of this result. First recall that 3-branes and 7-branes are very interesting for several reasons. Four-dimensional gauge theories with matter naturally exist with 3-branes and 7-branes, including the supersymmetric standard model [9]. The universe is automatically full of just the right ingredients to give the forces of nature. The large number of D3 branes could also provide a natural realization of AdS/CFT. This AdS space could play a critical role in localizing gravity 18] D3-branes and D7-branes are also important for recent ideas about string theory models of inflation 10, 11, 12. Recent string-theoretic inflation models are based on the presence of 3-branes and 7-branes [10, 11, 12]. As we will see shortly, this scenario could naturally give rise to this type of inflation.

The major issue we have left to address is the origin of four-dimensional gravity. We now suggest several reasons that four-dimensional gravity can exist, leaving the details for a forthcoming publication. Perhaps the least interesting possibility is that dimensions are in fact compact. One way our analysis could apply in that case is if nine spatial dimensions expand to a large size in the early evolution of the universe, and only afterwards stabilize at a small size. Or the universe gets effectively compactified without moduli fields due to the presence of the 7branes, which can effectively compactify dimensions as in Ref. 13]. 7-branes are codimension 2 branes in $10 \mathrm{~d}$ and as such they change the metric not just in their local neighborhood, but also affect the global geometry. For the generic 7-brane solution, for example the D7 brane of IIB string theory or a fat codimension 2-brane, as we will analyze below in the context of localized gravity, the metric naively becomes singular a finite distance away from the 7-brane. In order to see if the singularity can get resolved, one needs global information about the spacetime behind the singularity. In the case of $\mathrm{D} 7$ branes global solutions are known 13] in which for a finite number of 7-brane the space transverse to the 7-branes becomes compactified into a space that serves as the base of an elliptically fibered Calabi Yau. The geometry of the full Calabi-Yau encodes in addition the behavior of the scalar fields, the dilaton and the axion. The dimensionality of the compact space depends on the orientation of the 7branes. If all 7-branes are parallel, the transverse $2 \mathrm{~d}$ 
space becomes an $S^{2}$ base of an elliptically fibered K3. If they intersect over a $10-2 k$-dimensional spacetime, the transverse $2 k$-dimensional space has to be the base of a Calabi-Yau $(k+1)$-fold. This structure is governed by supersymmetry. It requires knowledge beyond the local form of the 7-brane metric. It is conceivable that in non-supersymmetric settings a globally consistent solution allows the local 7-brane geometry to be pasted into a compact space times a $4 \mathrm{~d}$ FRW part.

It is encouraging for this scenario that the recent string theory constructions of [11, 12] yielding compactifications with all moduli fixed and leading to quasi-realistic cosmologies rely on IIB compactification with 7-branes together with a huge number of 3-branes or fluxes carrying 3-brane charge. In these scenarios one starts out with the internal space being compact and only follows the cosmological evolution in the effective $4 \mathrm{~d}$ low energy theory. One can easily imagine this being the outcome of a 10d cosmological evolution that led 7- and 3-branes to dominate, presumably also determining which type of geometry is preferred. If for some reason the 3-branes tend to clump, in this case we can also get a scenario similar to that of Verlinde [14]. The 3-branes pull an $\mathrm{AdS}_{5} \times S^{5}$ throat out of the compact manifold, naturally stabilizing a large hierarchy.

However, perhaps the most promising scenario exploits the notion of localized gravity, which fits in naturally with our assumption that we can neglect compactification in the universe's early evolution. A configuration that is a natural candidate for four-dimensional gravity is the intersection of three 7 -branes where the intersection has spacetime dimension four. One can have a triple 7-brane intersection where the intersection has spacetime dimension four. It was shown in [15] that a single codimension 2-brane can localize gravity in an embedding AdS bulk space, so long as the brane satisfy a tuning conditions on its energy density. Using the methods of Ref. [16], one can generalize this setup to the case of 3 intersecting codimension 2 branes in $A d S_{10}$. There is a normalizable graviton and three-dimensional gravity localized at the interersection.

This shows one can consistently get three-dimensional gravity as well as forces localized on three branes in this scenario. The remaining challenge is to connect this configuration to the analysis of the first part of this paper; that is, to find a reason this configuration might be favored and to show why the forces should be coincident.

The problem is the intersections have a smaller filling fraction than the 7-branes themselves, or 5-brane intersections of two 7-branes for that matter. We suggest two reasons that the triple intersections might be favored, both of which merit further investigation. The first possibility is that the 3-branes play an important role in creating the intersection. That is, if there is an attractive force between 3-branes and 7-branes and they want to line up, this can create the preferred geometry. This would have the added feature of leading to gauge forces coincident with four-dimensional gravity. The second possibility that is under investigation is the possibility of four-dimensional "knots", which act as locally localized gravitational regions. The idea is that in addition to infinite 7-branes, there could be 7-brane loops in the directions orthogonal to the four overlapping dimensions on which gravity is localized. In order to localize gravity, the loops would probably have to have size somewhat bigger than the Planck length. They would therefore require flux passing through or on the branes themselves to stabilize them. The density of these finite-sized ocnfigurations (in extra-dimensional space) could be comparable to that of the fundamental 3-branes we considered earlier since from a cosmological perspective these 7 -brane balls look like 3-branes. Another advantage of these loop configurations might be that they could trap 3-branes in their interior, so that gravity and forces would be coincident. In fact, this raises the possibility of naturally explaining the hierarchy, since this would be the case if the 3-branes are near, but not precisely coincident, with the location where gravity peaks.

We also wish to mention the interesting inflationary scenario this model would suggest along the lines of [4, 17]. Even if branes are attracted to the triple intersection, it is likely that there is an initial excess of 3-branes or anti-3-branes, giving rise to a large cosmological energy density. Additional (anti-) 3 branes will aggregate at the intersection later on and annihilate as in 17]. Because the extra dimensions are big (in the second scenario) or infinite (in the first), one can naturally avoid some of the constraints that frustrated the initial brane-inflation, which doesn't last sufficiently long because the brane and antibrane cannot start off at large enough separation.

Acknowledgments: We would like to thank A. Albrecht, P. Creminelli, L. Fitzpatrick, L. Motl, A. Nelson, A. Nicolis, E. Silverstein, C. Vafa, and L. Wang for useful discussions, and S. Battersby for alerting us to the work of [3]. The work of AK was partially supported by the DOE under contract DE-FGO3-96-ER40956. The work of LR was supported in part by NSF Award PHY0201124 .
[1] R. H. Brandenberger and C. Vafa, Nucl. Phys. B316, 391 (1989).

[2] S. Alexander, R. H. Brandenberger, and D. Easson, Phys. Rev. D62, 103509 (2000), hep-th/0005212.

[3] R. Durrer, M. Kunz, and M. Sakellariadou (2005), hep- th/0501163.

[4] C. P. Burgess et al., JHEP 07, 047 (2001), hepth/0105204.

[5] S. Mukohyama and L. Randall, Phys. Rev. Lett. 92, 211302 (2004), hep-th/0306108. 
[6] J. Polchinski (2004), hep-th/0412244.

[7] R. Easther, B. R. Greene, M. G. Jackson, and D. Kabat, JCAP 0502, 009 (2005), hep-th/0409121.

[8] R. Danos, A. R. Frey, and A. Mazumdar, Phys. Rev. D70, 106010 (2004), hep-th/0409162.

[9] G. Aldazabal, L. E. Ibanez, F. Quevedo, and A. M. Uranga, JHEP 08, 002 (2000), hep-th/0005067.

[10] K. Dasgupta, C. Herdeiro, S. Hirano, and R. Kallosh, Phys. Rev. D65, 126002 (2002), hep-th/0203019.

[11] S. Kachru et al., JCAP 0310, 013 (2003), hepth/0308055.

[12] S. Kachru, R. Kallosh, A. Linde, and S. P. Trivedi, Phys. Rev. D68, 046005 (2003), hep-th/0301240.
[13] C. Vafa, Nucl. Phys. B469, 403 (1996), hep-th/9602022.

[14] I. Savonije and E. Verlinde, Phys. Lett. B507, 305 (2001), hep-th/0102042.

[15] T. Gherghetta and M. E. Shaposhnikov, Phys. Rev. Lett. 85, 240 (2000), hep-th/0004014.

[16] N. Arkani-Hamed, S. Dimopoulos, G. R. Dvali, and N. Kaloper, Phys. Rev. Lett. 84, 586 (2000), hepth/9907209.

[17] G. R. Dvali and S. H. H. Tye, Phys. Lett. B450, 72 (1999), hep-ph/9812483.

[18] We thank Bobby Acharya for suggesting an interesting model of this sort. 